\title{
Hot topics in epigenetic regulation of cancer self-renewal for pancreatic tumors: future trends
}

\author{
Ramon Andrade De Mello*,1,2,3,4, Inês Faleiro',4, Joana D Apolónio ${ }^{1,4}$, Uri Tabori ${ }^{5}$, Aryeh J \\ Price $^{5,6}$, Vânia P Roberto ${ }^{1,4}$ \& Pedro Castelo-Branco ${ }^{1,4}$ \\ ${ }^{1}$ Department of Biomedical Sciences \& Medicine, Algarve Biomedical Center, Division of Oncology, University of Algarve, 8005-139 \\ Faro, Portugal \\ ${ }^{2}$ Faculty of Medicine, University of Porto, 4200-319, Oporto, Portugal \\ ${ }^{3}$ Division of Medical Oncology, Bauru State Hospital, \& School of Medicine, Universidade Nove de Julho (UNINOVE), \\ 17011-102, Bauru, São Paulo, Brazil \\ ${ }^{4}$ Centre for Biomedical Research (CBMR), University of Algarve, 8005-139 Faro, Portugal \\ ${ }^{5}$ Arthur \& Sonia Labatt Brain Tumor Research Center, The Hospital for Sick Children, University of Toronto, Toronto, M5G 1X8, ON, \\ Canada \\ ${ }^{6}$ Division of Biology \& Biological Engineering, California Institute of Technology, Pasadena, CA 91125, USA \\ *Author for correspondence: ramondemello@gmail.com
}

\begin{abstract}
"Pancreatic cancer is both a deadly and highly recurrent tumor, relying heavily on accelerated proliferation. Understanding self-renewal in this tumor type is therefore likely to prove essential for reaching better disease control."”
\end{abstract}

First draft submitted: 24 February 2018; Accepted for publication: 19 December 2018; Published online: 29 January 2019

Keywords: biomarkers $\bullet$ gastrointestinal $\bullet$ molecular oncology $\bullet$ oncogenes $\bullet$ pancreatic-biliary $\bullet$ THOR

Self-renewal is critical in order for a cancer to proliferate, grow and eventually metastasize. Today, several molecular mechanisms are being studied, which may explain the emergence of this property in advanced cancer. Pancreatic cancer is both a deadly and highly recurrent tumor, relying heavily on accelerated proliferation. Understanding self-renewal in this tumor type is therefore likely to prove essential for reaching better disease control. Recent advances in our understanding of telomere maintenance pathways are beginning to yield those insights [1]. Indeed, it is now known that telomerase reactivation in cancer is intimately related with expression of the TERT gene. The multimodal regulation of this locus, along with transcriptional output itself, both serve as prognostic factors across a variety of tumor types.

In 2006, Clément et al. analyzed early molecular markers of malignant transformation that have the potential for clinical implementation [2]. Gene promoter methylation analysis was performed on normal, preneoplastic and neoplastic lesions from Barrett's esophagus (BE) patients. The TERT promoter was hypermethylated in 100, 91 and $92 \%$ of cases, whereas in BE mucosa from patients who had not progressed (16 samples) methylation was found only in 36, 23 and 17\%, respectively. These data suggested that promoter methylation profiling of BE using multiple target genes including APC, TIMP3 and TERT could be used as a predictive panel for increased esophageal adenocarcinoma susceptibility [2].

In 2013, Castelo-Branco et al. suggested that hypermethylation of a specific region in the TERT promoter is associated with TERT expression. The signature discovered is predictive of tumor progression and poor prognosis in pediatric brain tumors. This differentially methylated region is remarkably accessible, given that it is easy to amplify, and readily assayed using standard clinical laboratory reagents [3]. Later studies have supported pan-cancer significance for this biomarker, including a correlation with biochemical recurrence in prostate cancer [4] and diagnostic capacity in neuroblastoma [5].

Pancreatic cancer proved to be an excellent application for this new biomarker. Recently, we analyzed data retrieved from the The Cancer Genome Atlas (TCGA; http://xena.ucsc.edu/) on 193 patients with pancreatic adenocarcinoma [1]. Exclusion criteria included history of neoadjuvant radiotherapy or chemotherapy as those

Future $\because \cdots$ Medicine 
parameters could independently influence methylation levels. We used the Illumina Infinium HumanMethylation $450 \mathrm{~K}$ array to analyze this cohort (normal tissue $[\mathrm{n}=10]$ and primary tumor $[\mathrm{n}=183]$ ). We found the CpG site targeted by the probe cg11625005 (chr5:1,295,737; position -575 in relation to transcription start site), localized within the TERT hypermethylated oncologic region (THOR; -591/-159), in pancreatic adenocarcinoma. Since THOR was shown to associate with TERT expression [3], our group investigated TERT expression in pancreatic cancer and found a positive correlation with THOR methylation levels. In addition, THOR could differentiate normal tissue from stage I to IV of the disease supporting its use as an early disease biomarker.

Currently, advanced pancreatic cancer approaches are very limited in terms of overall survival benefits [6]. Finding a novel biomarker for tailoring treatment is an urgent unmet clinical need. Extant biomarkers are generally classical precursors of pancreatic cancer, such as KRAS mutations and inactivation of tumor suppressor genes (TP53, $p 16 / C D K N 2 A$ and SMAD4) $[7,8]$. Improving on the status quo and finding meaningful biomarkers will require developing a deep understanding of pancreatic cancer pathogenesis. THOR is one such example. Highly methylated THOR correlates with overall and recurrence-free survival of the patients suggesting that THOR can help predict patient outcome. In borderline resectable disease, we hypothesize that THOR data could help clinicians decide whether or not to indicate chemotherapy followed by radiation and then surgery or refer the patient for a clinical trial. In selected patients with ECOG performance status 2 due to heavy tumor load, THOR levels might identify the optimal chemotherapy regimen for achieving a response. There are many more clinical prospects for THOR, and biomarkers in its class; however, larger cohorts and randomized Phase III trials will be necessary.

Furthermore, circulating tumors cells (CTCs) faces an interesting scenario in the clinical practice [9]. Today, several platforms and methods have been described to detect and isolate CTCs from the human blood [9-11]. The liquid biopsy, the detection and enumerating of CTCs, is considered a very practical and useful clinical tool to monitoring treatment responses and estimate the probability of tumor relapse. Also, it is an important noninvasive tool for molecular characterization and tumor biomarkers analysis without need of tumor tissue biopsies. In 2015, Salanti et al. reported an interesting research [12], which showed the role of VAR2CSA malaria protein for the oncofetal chondroitin sulfate (ofCS) detection, a highly specific target for CTCs isolation. The ofCS is expressed by placental and cancer cells of both epithelial and mesenchymal origin [13]. The authors [12] suggested that ofCS could play a key role in tumor-cell motility through integrin signaling pathways, and therefore supports the metastatic potential of cancer cells [13]. Testing a wide range of cells and tissues, the authors found that the recombinant VAR2CSA protein binds $>95 \%$ of cancer cell lines and tissues of epithelial, mesenchymal and hematopoietic origin, with very limited binding to noncancerous cells or normal tissue (besides placental tissue), which it could improve its role as tool to broadly and efficiently capture rare cancer cells in complex blood samples [10,12]. Liquid biopsy is approved by the US FDA for the EGFR analysis in lung cancer patients [14]. Currently, the CellSearch ${ }^{T M}$ platform is the only gold standard and approved by FDA for all the CTCs detection strategies [14]. In addition, methylation analysis of circulating tumor DNA could be useful to determination of the primary cancer location with a specificity and sensitivity of 99.6 and $97.7 \%$, respectively $[15,16]$. It would be a very helpful tool in the near future to characterize pancreatic cancer molecular profile in order to help clinicians in terms of personalized medicine. Thus, more robust and well-designed clinical trials are important to assess the role of all these aforementioned biomarkers in the pancreatic cancer framework.

Financial \& competing interests disclosure

RA De Mello is on advisory board/speaker for MSD, Pfizer, Zodiac, Astrazeneca and Novartis. The study received research grant N $^{\circ}$ 402621/2016-6 from (National Research Council) Conselho Nacional de Desenvolvimento Científico e Tecnológico (CNPQ) Brazil, Roche and Merck Group. The study has received travel grant from Roche, BMS, Pierre Fabre and Amgen. JD Apolónio and VP Roberto were the recipients of PD/BD/105899/2014 FCT and LPCC-Fundação PT2016 fellowships, respectively. The authors have no other relevant affiliations or financial involvement with any organization or entity with a financial interest in or financial conflict with the subject matter or materials discussed in the manuscript apart from those disclosed.

No writing assistance was utilized in the production of this manuscript.

\section{References}

Papers of special note have been highlighted as: $\bullet$ of interest; $\bullet \bullet$ of considerable interest

1. Faleiro I, Apolónio JD, Price AJ et al. The TERT hypermethylated oncologic region predicts recurrence and survival in pancreatic cancer. Future Oncol. 13(23) 2045-2051 (2017).

- Important paper, which shows the role of TERT hypermethylated oncologic region in pancreatic cancer aggressiveness. 
2. Clément G, Braunschweig R, Pasquier N et al. Methylation of APC, TIMP3, and TERT: a new predictive marker to distinguish Barrett's oesophagus patients at risk for malignant transformation. J. Pathol. 208(1), 100-107 (2006).

3. Castelo-Branco P, Choufani S, Mack S et al. Methylation of the TERT promoter and risk stratification of childhood brain tumours: an integrative genomic and molecular study. Lancet Oncol. 14(6), 534-542 (2013).

-. Important paper, which addresses the role of TERT hypermethylated oncologic region in brain tumors.

4. Castelo-Branco P, Leão R, Lipman T et al. A cancer specific hypermethylation signature of the TERT promoter predicts biochemical relapse in prostate cancer: a retrospective cohort study. Oncotarget 7(36), 57726 (2016).

5. Lee S, Borah S, Bahrami A. Detection of aberrant TERT promoter methylation by combined bisulfite restriction enzyme analysis for cancer diagnosis. J. Mol. Diagn. 19(3), 378-386 (2017).

6. Ducreux M, Caramella C, Hollebecque A et al. Cancer of the pancreas: ESMO clinical practice guidelines for diagnosis, treatment and follow-up. Ann. Oncol. 26(Suppl. 5), v56-v68 (2015).

7. Waddell N, Pajic M, Patch A-M et al. Whole genomes redefine the mutational landscape of pancreatic cancer. Nature 518(7540), 495 (2015).

8. de Mello RA, Pousa I, Pereira D. Nivolumab for advanced squamous cell lung cancer: what are the next steps? Lancet Oncol. 16(3), 234-235 (2015).

9. DiPardo BJ, Winograd P, Court CM et al. Pancreatic cancer circulating tumor cells: applications for personalized oncology. Expert Rev. Mol. Diagn.18(9), 809-820 (2018).

10. Parkinson DR, Dracopoli N, Petty BG et al. Considerations in the development of circulating tumor cell technology for clinical use. J. Transl. Med. 10(1), 138 (2012).

11. Yu M, Stott S, Toner M et al. Circulating tumor cells: approaches to isolation and characterization. J. Cell Biol. 192(3), 373-382 (2011).

12. Salanti A, Clausen TM, Agerbæk MØ et al. Targeting human cancer by a glycosaminoglycan binding malaria protein. Cancer Cell 28(4), 500-514 (2015).

13. Agerbæk MØ, Bang-Christensen SR, Yang M-H et al. The VAR2CSA malaria protein efficiently retrieves circulating tumor cells in an EpCAM-independent manner. Nat. Commun. 9(1), 3279 (2018).

14. Kuske A, Gorges TM, Tennstedt P et al. Improved detection of circulating tumor cells in non-metastatic high-risk prostate cancer patients. Sci. Rep. 6, 39736 (2016).

15. Moran S, Martínez-Cardús A, Sayols S et al. Epigenetic profiling to classify cancer of unknown primary: a multicentre, retrospective analysis. Lancet Oncol. 17(10), 1386-1395 (2016).

16. Yadav DK, Bai X, Yadav RK et al. Liquid biopsy in pancreatic cancer: the beginning of a new era. Oncotarget 9(42), 26900 (2018). 
